\title{
LOG-BALANCED COMBINATORIAL SEQUENCES
}

\author{
TOMISLAV DOŠLIĆ
}

Received 25 July 2004 and in revised form 9 December 2004

We consider log-convex sequences that satisfy an additional constraint imposed on their rate of growth. We call such sequences log-balanced. It is shown that all such sequences satisfy a pair of double inequalities. Sufficient conditions for log-balancedness are given for the case when the sequence satisfies a two- (or more-) term linear recurrence. It is shown that many combinatorially interesting sequences belong to this class, and, as a consequence, that the above-mentioned double inequalities are valid for all of them.

\section{Introduction}

One of the most common tasks in combinatorics is to find explicitly the size of a certain finite set, depending on an integer parameter $n$ and defined in an intricate way. Then the next question usually asks how the sequence of numbers describing this size behaves for large values of $n$. Of particular interest is logarithmic behavior of the sequence (i.e., its log-convexity or log-concavity), since it is often instrumental in obtaining its growth rate and asymptotic behavior. Also, log-behavior may qualify (or disqualify) a sequence as a candidate for use in certain models. A good example is the recent use of log-convex sequences in quantum physics for constructing generalized coherent states associated with models having discrete nonlinear spectra [12].

The literature on log-behavior of combinatorial sequences is vast; we refer the reader to the book [10], and also to $[5,14,16]$.

In this paper, we quantitatively refine the concept of log-convexity by introducing and considering the class of log-balanced combinatorial sequences and showing that the terms of such sequences satisfy certain double inequalities. We further proceed by deriving sufficient conditions for a (combinatorial) sequence given by a two-term linear homogeneous recurrence to be log-convex and log-balanced. We also indicate how to extend this approach to longer recurrences and how to treat the case of nonhomogeneous recurrences. Finally, we demonstrate that the class of log-balanced sequences is rich enough to include many cases of special combinatorial interest. As a consequence, we obtain new pairs of inequalities for many classical sequences. 


\section{Log-balanced sequences}

A sequence $\left(a_{n}\right)_{n \geq 0}$ of positive real numbers is log-convex if $a_{n}^{2} \leq a_{n-1} a_{n+1}$ for all $n \geq 1$. If the opposite inequality $a_{n}^{2} \geq a_{n-1} a_{n+1}$ is valid for all $n \geq 1$, we say that the sequence $\left(a_{n}\right)_{n \geq 0}$ is $\log$-concave. In case of equality, $a_{n}^{2}=a_{n-1} a_{n+1}, n \geq 1$, we call the sequence $\left(a_{n}\right)_{n \geq 0}$ geometric or log-straight. Another type of logarithmic behavior is that of the Fibonacci sequence, where the direction of the inequality depends on the parity of $n$. We call such sequences log-Fibonacci.

An alternative way of characterizing the log-behavior of a sequence is via the sequence of quotients of its successive terms. We call the sequence $\left(x_{n}\right)_{n \geq 1}, x_{n}=a_{n} / a_{n-1}$ the quotient sequence of the sequence $\left(a_{n}\right)_{n \geq 0}$. Obviously, the sequence $\left(a_{n}\right)_{n \geq 0}$ is log-convex if and only if its quotient sequence is nondecreasing. Similarly, $\left(a_{n}\right)_{n \geq 0}$ is log-concave if and only if its quotient sequence is nonincreasing, and log-Fibonacci if and only if no three successive elements of the quotient sequence form a monotone subsequence.

In what follows, we consider log-convex sequences whose quotient sequence does not grow too fast. We will also assume that $a_{0}=1$, unless explicitly stated otherwise. This restriction is not too severe, since in many combinatorially interesting cases we put $a_{0}=1$ by convention.

A sequence $\left(a_{n}\right)_{n \geq 0}$ of positive real numbers is log-balanced if $\left(a_{n}\right)_{n \geq 0}$ is log-convex and the sequence $\left(a_{n} / n !\right)_{n \geq 0}$ is log-concave. In terms of quotient sequences, this means that $x_{n} \leq x_{n+1} \leq((n+1) / n) x_{n}$, for all $n \geq 1$.

The motivation for considering such sequences comes from the recent paper [2], where it was shown that the sequences of Bell numbers of any order are of this type. Since this property makes them suitable for providing important examples in white noise theory [11], it is of interest to see whether there are some other such sequences and to characterize them.

We start by stating in terms of log-balanced sequences the following observation, made in [2]. The proof is reproduced here for the reader's convenience.

Proposition 2.1. Let $\left(a_{n}\right)_{n \geq 0}$ be a log-balanced sequence. Then

(a) $a_{n}^{2} \leq a_{n-1} a_{n+1} \leq(1+1 / n) a_{n}^{2}, n \geq 1$;

(b) $a_{n} a_{m} \leq a_{n+m} \leq\left(\begin{array}{c}n+m \\ n\end{array}\right) a_{n} a_{m}, n, m \geq 0$.

Proof. The double inequality (a) is just another way of stating the fact that the sequence $\left(a_{n}\right)_{n \geq 0}$ is log-balanced.

The left inequality of (b) follows easily (by induction) from the log-convexity of $\left(a_{n}\right)_{n \geq 0}$. To prove the right inequality, start from $x_{n} \geq(n /(n+1)) x_{n+1}$. By using this inequality repeatedly, we get

$$
\frac{a_{1}}{a_{0}} \geq \frac{1}{2} \frac{a_{2}}{a_{1}} \geq \frac{1}{3} \frac{a_{3}}{a_{2}} \geq \cdots \geq \frac{1}{m+n} \frac{a_{m+n}}{a_{m+n-1}},
$$

for all $n \geq 0, m \geq 1$.

Hence, for any $0 \leq j \leq m-1$, we have

$$
\frac{a_{j+1}}{a_{j}} \geq \frac{j+1}{m+n} \frac{a_{m+n}}{a_{m+n-1}} .
$$


From this we get

$$
\frac{a_{1}}{a_{0}} \frac{a_{2}}{a_{1}} \frac{a_{3}}{a_{2}} \cdots \frac{a_{m}}{a_{m-1}} \geq\left(\frac{1}{n+1} \frac{a_{n+1}}{a_{n}}\right)\left(\frac{2}{n+2} \frac{a_{n+2}}{a_{n+1}}\right) \cdots\left(\frac{m}{m+n} \frac{a_{m+n}}{a_{n}}\right) .
$$

After the cancellations we get

$$
\frac{a_{m}}{a_{0}} \geq \frac{n ! m !}{(m+n) !} \frac{a_{m+n}}{a_{n}}
$$

and, taking into account the fact that $a_{0}=1$, we finally get

$$
a_{m+n} \leq\left(\begin{array}{c}
m+n \\
n
\end{array}\right) a_{n} a_{m} .
$$

The case $m=0$ is trivially valid for all $n \geq 0$.

\section{Sufficient conditions}

For most sequences of combinatorial interest there are no explicit, closed form expressions for their elements. On the other hand, one can often find recurrences and/or generating functions for them. So, direct ways of establishing the log-behavior of a given sequence (i.e., of proving inequalities of the type (a) from Proposition 2.1) are only rarely at our disposal. Combinatorial proofs, which are the most desirable, often turn out to be rather involved and/or tricky. (A nice survey of inductive and injective proofs of logconcavity is given in [13].) Hence, it makes sense to seek analytical methods sufficiently robust and easy to apply, and that will work for a reasonably broad class of sequences. Here we present one such method that works almost automatically for sequences given by recurrence relations. We start by explaining the method for the case of linear homogeneous recurrences of second order, and later we indicate how to modify this so that it can be applied also on longer and/or nonhomogeneous recurrences.

Let $\left(a_{n}\right)_{n \geq 0}$ be a sequence of positive real numbers, given by the two-term recurrence

$$
a_{n}=R(n) a_{n-1}+S(n) a_{n-2}, \quad n \geq 2,
$$

with given initial conditions $a_{0}, a_{1}$. The quotient sequence $\left(x_{n}\right)_{n \geq 1}$ satisfies the nonlinear recurrence

$$
x_{n}=R(n)+\frac{S(n)}{x_{n-1}}, \quad n \geq 2,
$$

with the initial condition $x_{1}=a_{1} / a_{0}$. We assume that the sequence $\left(x_{n}\right)$ is bounded by two known sequences, that is, that there are sequences $\left(m_{n}\right)$ and $\left(M_{n}\right)$ such that $0<m_{n} \leq x_{n} \leq$ $M_{n}$, for all $n \in \mathbb{N}$. The sequences $\left(m_{n}\right)$ and $\left(M_{n}\right)$ can usually be rather easily inferred from recurrence (3.2), or guessed from the initial behavior of the sequence $\left(x_{n}\right)$, and then the bounding relations are verified by induction. In many cases even the constant sequences $m_{n}=m$ and $M_{n}=M$ will be sufficiently good lower and upper bounds for $x_{n}$.

As the log-convexity is of considerable interest on its own, we first establish sufficient conditions for a sequence $\left(a_{n}\right)$ given by (3.1) to be log-convex. We assume that $R(n) \geq 0$ 
and treat the cases $S(n) \leq 0$ and $S(n) \geq 0$ separately. The case $S(n) \leq 0$ is simpler and we consider it first.

Assume, inductively, that $x_{n_{0}} \leq x_{n_{0}+1} \leq \cdots \leq x_{n}$ for some $n_{0} \in \mathbb{N}$. Expressing $x_{n+1}$ from (3.2) and taking into account that $S(n+1) / x_{n} \geq S(n+1) / x_{n-1}$, we obtain

$$
x_{n+1}=R(n+1)+\frac{S(n+1)}{x_{n}} \geq R(n+1)+\frac{S(n+1)}{x_{n-1}} .
$$

We want to prove that $x_{n+1} \geq x_{n}$. But this will follow if we prove the stronger inequality in which $x_{n+1}$ is replaced by the right-hand side in the above inequality. Hence, consider the circumstance

$$
R(n+1)+\frac{S(n+1)}{x_{n-1}} \geq x_{n}=R(n)+\frac{S(n)}{x_{n-1}},
$$

or, equivalently,

$$
[R(n+1)-R(n)] x_{n-1}+S(n+1)-S(n) \geq 0 .
$$

By denoting $R(n+1)-R(n)=\nabla R(n)$ and $S(n+1)-S(n)=\nabla S(n)$, we get a compact expression for the sufficient condition for the sequence $\left(a_{n}\right)$ to be log-convex:

$$
\nabla R(n) x_{n-1}+\nabla S(n) \geq 0, \quad n \geq n_{0}
$$

for some $n_{0} \in \mathbb{N}$. Hence, we have established the following result.

Proposition 3.1. Let $\left(a_{n}\right)_{n \geq 0}$ be a sequence of positive real numbers given by the two-term recurrence (3.1), and $\left(x_{n}\right)_{n \geq 1}$ its quotient sequence, given by (3.2). If there is an $n_{0} \in \mathbb{N}$ such that $x_{n_{0}} \leq x_{n_{0}+1}, R(n) \geq 0, S(n) \leq 0$, and

$$
\nabla R(n) x_{n-1}+\nabla S(n) \geq 0
$$

for all $n \geq n_{0}$, then the sequence $\left(a_{n}\right)_{n \geq n_{0}}$ is log-convex.

When (as is a common case) the function $R(n)$ is nondecreasing, the condition (3.6) can be further simplified without significant loss of generality by assuming $\nabla R(n) \geq 0$ and replacing $x_{n-1}$ by $m_{n-1}$, or even by a constant $m$ :

$$
\nabla R(n) m+\nabla S(n) \geq 0, \quad n \geq n_{0}
$$

The case $S(n) \geq 0$ is a bit more complicated. Again, we start from the inductive assumption $x_{n_{0}} \leq x_{n_{0}+1} \leq \cdots \leq x_{n}$ and want to show that $x_{n+1} \geq x_{n}$. By expressing both sides of this inequality via (3.2), we obtain

$$
R(n+1)+\frac{S(n+1)}{x_{n}} \geq R(n)+\frac{S(n)}{x_{n-1}} .
$$


This is equivalent to

$$
x_{n} x_{n-1} \nabla R(n)+S(n+1) x_{n-1}-S(n) x_{n} \geq 0 .
$$

By adding the term $S(n) x_{n-1}-S(n) x_{n-1}$ to the left-hand side of the above inequality and rearranging it, we obtain

$$
\nabla R(n) x_{n} x_{n-1}+\nabla S(n) x_{n-1} \geq S(n)\left(x_{n}-x_{n-1}\right) .
$$

Expressing the term $x_{n}-x_{n-1}$ via (3.2) now yields

$$
x_{n-1}\left[\nabla R(n) x_{n}+\nabla S(n)\right] \geq S(n)\left[\nabla R(n-1)+\frac{S(n)}{x_{n-1}}-\frac{S(n-1)}{x_{n-2}}\right] .
$$

Now, replacing $S(n) / x_{n-1}$ with $S(n) / x_{n-2}$ in the right-hand side square brackets, we get a stronger inequality which can be written as

$$
x_{n-1} x_{n-2}\left[\nabla R(n) x_{n}+\nabla S(n)\right] \geq S(n)\left[\nabla R(n-1) x_{n-2}+\nabla S(n-1)\right]
$$

Obviously, this inequality implies $x_{n+1} \geq x_{n}$, and it can serve as a sufficient condition of log-convexity for the sequence $\left(a_{n}\right)$.

Proposition 3.2. Let $\left(a_{n}\right)_{n \geq 0}$ be a sequence of positive real numbers given by the two-term recurrence (3.1), and $\left(x_{n}\right)_{n \geq 1}$ its quotient sequence, given by (3.2). If there is an $n_{0} \in \mathbb{N}$ such that $x_{n_{0}} \leq x_{n_{0}+1}, R(n) \geq 0, S(n) \geq 0$, and the inequality

$$
x_{n-1} x_{n-2}\left[\nabla R(n) x_{n}+\nabla S(n)\right] \geq S(n)\left[\nabla R(n-1) x_{n-2}+\nabla S(n-1)\right]
$$

is valid for all $n \geq n_{0}$, then the sequence $\left(a_{n}\right)_{n \geq n_{0}}$ is log-convex.

Again, in many combinatorially relevant cases where $\nabla R(n) \geq 0$ and $m \leq x_{n} \leq M$, the sufficient condition of Proposition 3.2 can be simplified to

$$
m^{2}[m \nabla R(n)+\nabla S(n)] \geq S(n)[M \nabla R(n-1)+\nabla S(n-1)] .
$$

Typically, Propositions 2.1 and/or 3.1 are applied so that the respective inequalities are verified inductively for all $n \in \mathbb{N}$ greater than some $n_{0}$, and the remaining cases are then checked by hand or using some computer algebra system.

Now we turn our attention to the inequality $x_{n+1} \leq((n+1) / n) x_{n}$. Again, we assume $R(n) \geq 0$ and treat the cases $S(n) \leq 0$ and $S(n) \geq 0$ separately. Also, we assume that the log-convexity of the sequence $\left(a_{n}\right)$ is already established, that is, that the sequence $\left(x_{n}\right)$ is increasing.

We first consider the simpler case $R(n) \geq 0, S(n) \geq 0$ and find the sufficient conditions for $x_{n+1} \leq((n+1) / n) x_{n}$ as follows. From the recurrence (3.2) we have

$$
x_{n+1}=R(n+1)+\frac{S(n+1)}{x_{n}} .
$$


512 Log-balanced combinatorial sequences

Since the sequence $\left(x_{n}\right)_{n \geq 1}$ is nondecreasing, we have

$$
x_{n+1} \leq R(n+1)+\frac{S(n+1)}{x_{n-1}} .
$$

The condition that the right-hand side does not exceed $((n+1) / n) x_{n}$ is given by

$$
R(n+1)+\frac{S(n+1)}{x_{n-1}} \leq \frac{n+1}{n}\left(R(n)+\frac{S(n)}{x_{n-1}}\right)
$$

and this is equivalent to

$$
n R(n+1) x_{n-1}+n S(n+1) \leq(n+1) R(n) x_{n-1}+(n+1) S(n)
$$

Denoting

$$
\Delta_{R}(n)=\left|\begin{array}{cc}
R(n) & n \\
R(n+1) & n+1
\end{array}\right|, \quad \Delta_{S}(n)=\left|\begin{array}{cc}
S(n) & n \\
S(n+1) & n+1
\end{array}\right|
$$

we get our sufficient conditions in the form

$$
\Delta_{R}(n) x_{n-1}+\Delta_{S}(n) \geq 0
$$

Hence, we have established the following result.

Proposition 3.3. Let $\left(a_{n}\right)_{n \geq 0}$ be a log-convex sequence of positive real numbers given by the two-term recurrence (3.1). If there is an $n_{0} \in \mathbb{N}$ such that $x_{n_{0}+1} \leq\left(\left(n_{0}+1\right) / n_{0}\right) x_{n_{0}}$, $R(n) \geq 0, S(n) \geq 0$, and

$$
\Delta_{R}(n) x_{n-1}+\Delta_{S}(n) \geq 0
$$

for all $n \geq n_{0}$, then the sequence $\left(a_{n}\right)_{n \geq 0}$ is log-balanced.

The case $S(n) \leq 0$ is a bit more complicated. We proceed by induction on $n$. First we check that $x_{n_{0}+1} \leq\left(\left(n_{0}+1\right) / n_{0}\right) x_{n_{0}}$ for some $n_{0} \in \mathbb{N}$, and suppose that $x_{k} \leq(k /(k-1)) x_{k-1}$ for all $n_{0} \leq k \leq n$. Denoting $-S(n)=\widetilde{S}(n)$, we get

$$
x_{n+1}=R(n+1)-\frac{\tilde{S}(n+1)}{x_{n}}, \quad \tilde{S}(n+1) \geq 0 .
$$

From the induction hypothesis, $x_{n} \leq(n /(n-1)) x_{n-1}$, it follows that $1 / x_{n} \geq((n-1) /$ $n)\left(1 / x_{n-1}\right)$, and hence $-1 / x_{n} \leq-((n-1) / n)\left(1 / x_{n-1}\right)$. Now we have

$$
x_{n+1}=R(n+1)-\frac{\widetilde{S}(n+1)}{x_{n}} \leq R(n+1)-\frac{n-1}{n} \frac{\widetilde{S}(n+1)}{x_{n-1}} \text {. }
$$


The right-hand side does not exceed $((n+1) / n) x_{n}$ if

$$
R(n+1)-\frac{n-1}{n} \frac{\widetilde{S}(n+1)}{x_{n-1}} \leq \frac{n+1}{n}\left(R(n)-\frac{\tilde{S}(n)}{x_{n-1}}\right)
$$

and this is, in turn, equivalent to

$$
[(n+1) R(n)-n R(n+1)] x_{n-1}+(n-1) \tilde{S}(n+1)-(n+1) \tilde{S}(n) \geq 0 .
$$

The coefficient of $x_{n-1}$ is $\Delta_{R}(n)$, and the rest can be written as

$$
\left|\begin{array}{cc}
n-1 & \widetilde{S}(n) \\
n+1 & \widetilde{S}(n+1)
\end{array}\right|=\left|\begin{array}{cc}
S(n) & n-1 \\
S(n+1) & n+1
\end{array}\right| .
$$

Denoting the right-hand side determinant by $\bar{\Delta}_{S}(n)$, we get the desired sufficient conditions:

$$
\Delta_{R}(n) x_{n-1}+\bar{\Delta}_{S}(n) \geq 0
$$

We can summarize.

Proposition 3.4. Let $\left(a_{n}\right)_{n \geq 0}$ be a log-convex sequence of positive real numbers given by the two-term recurrence (3.1) with $R(n) \geq 0, S(n) \leq 0$. If there is an integer $n_{0}$ such that $x_{n_{0}+1} \leq\left(\left(n_{0}+1\right) / n_{0}\right) x_{n_{0}}$, and if the inequality

$$
\Delta_{R}(n) x_{n-1}+\bar{\Delta}_{S}(n) \geq 0
$$

holds for all $n \geq n_{0}$, then the sequence $\left(a_{n}\right)_{n \geq n_{0}}$ is log-balanced.

\section{Examples}

We now justify our introduction of log-balanced sequences by demonstrating that the class is wide enough and that it includes many sequences of combinatorial relevance. As a consequence, for all our examples we establish the validity of inequalities from Proposition 2.1. The left inequalities for some of the considered sequences were established earlier $[1,9]$, but the right inequalities are, with one exception [8], to the best of our knowledge, new. For more details on all the considered sequences, we refer the reader to the book [15] and to the references therein.

Our first example is the sequence of Motzkin numbers (see, e.g., [15, Exercise 6.38] for its combinatorial interpretations).

Corollary 4.1. The sequence $M_{n}$ of Motzkin numbers is log-balanced.

Proof. The log-convexity of $M_{n}$ was first established algebraically in [1], and a combinatorial proof appeared soon afterwards [6]. By our method it follows easily by starting 
from the recurrence

$$
M_{n}=\frac{2 n+1}{n+2} M_{n-1}+\frac{3(n-1)}{n+2} M_{n-2}, \quad n \geq 2,
$$

with $M_{0}=M_{1}=1$. Here $R(n)=(2 n+1) /(n+2) \geq 0, S(n)=3(n-1) /(n+2) \geq 0$. It is easy to prove by induction on $n$ that $2 \leq M_{n} / M_{n-1} \leq 7 / 2$ for all $n \geq 2$, and the logconvexity follows by computing $\nabla R(n), \nabla S(n), \nabla R(n-1)$, and $\nabla S(n-1)$ and then verifying the inequality (3.15). From the fact that $\Delta_{R}(n)=\left(2 n^{2}+4 n+3\right) /(n+2)(n+3) \geq 0$, $\Delta_{S}(n)=\left(n^{2}-n-3\right) /(n+2)(n+3) \geq 0$, and $x_{n-1} \geq 0$ for all $n \geq 3$, it follows that $\Delta_{R}(n) x_{n-1}+\Delta_{S}(n) \geq 0$ for all $n \geq 3$. The log-balancedness of $\left(M_{n}\right)$ now follows from Proposition 3.3, after direct verification of the defining inequality for the remaining values of $n$.

Our next example is the sequence of Fine numbers. The reader may consult the recent survey [7] for more details on Fine numbers and on their combinatorial interpretations.

Corollary 4.2. The sequence $B_{n}$ of Fine numbers is log-balanced for $n \geq 2$.

Proof. We start from the recurrence

$$
B_{n}=\frac{7 n-5}{2 n+2} B_{n-1}+\frac{2 n-1}{n+1} B_{n-2}, \quad n \geq 2,
$$

with initial conditions $B_{0}=1$ and $B_{1}=0$. The quotient sequence, $x_{n}=B_{n} / B_{n-1}$, is defined for $n \geq 3$. It is easy to show, by induction on $n$, that $3 \leq x_{n} \leq 6$ for all $n \geq 3$. In fact, $3 \leq x_{n-1} \leq 6$ implies $3 \leq x_{n} \leq 6$ via the above recurrence for $n \geq 7$, and $x_{n}$ is obviously between 3 and 6 for $n=3,4,5$, and 6 . We proceed by computing $\nabla R(n)=6 /(n+1)(n+2)$, $\nabla S(n)=4 /(n+1)(n+2), \nabla R(n-1)=6 / n(n+1)$, and $\nabla S(n-1)=4 / n(n+1)$. After plugging in these expressions, condition (3.15) becomes

$$
10 n^{2}-30 n+80 \geq 0
$$

and this is true for all $n \in \mathbb{N}$. Hence, the sequence $\left(B_{n}\right)_{n \geq 2}$ is log-convex. The logbalancedness now follows by computing $\Delta_{R}(n)=(7 n-10) / 2(n+2), \Delta_{S}(n)=2((n-1) /$ $(n+2))$, and applying Proposition 3.3.

The Franel numbers of order $r$ are defined by

$$
F_{n}^{(r)}=\sum_{k=0}^{n}\left(\begin{array}{l}
n \\
k
\end{array}\right)^{r}
$$

Corollary 4.3. The sequences of Franel numbers of order 3 and 4 are log-balanced.

Proof. It is known that Franel numbers of order $r$ satisfy a homogeneous linear recurrence of order $\lfloor(r+1) / 2\rfloor$ with polynomial coefficients (see [15, pages 245-246 and page 278]). We have

$$
F_{n}^{(r)}=R^{(r)}(n) F_{n-1}^{(r)}+S^{(r)}(n) F_{n-2}^{(r)}, \quad r=3,4, n \geq 2,
$$


with $F_{0}^{(3)}=F_{0}^{(4)}=1, F_{1}^{(3)}=F_{1}^{(4)}=2$. Here

$$
\begin{array}{ll}
R^{(3)}(n)=\frac{7 n^{2}-7 n+2}{n^{2}}, & S^{(3)}(n)=\frac{8(n-1)^{2}}{n^{2}}, \\
R^{(4)}(n)=2 \frac{6 n^{3}-9 n^{2}+5 n-1}{n^{3}}, & S^{(4)}(n)=\frac{(4 n-3)(4 n-4)(4 n-5)}{n^{3}} .
\end{array}
$$

Obviously, all coefficient functions are nonnegative. We work out the case $r=3$ and leave the details for $r=4$ to the interested reader. By examining first few values of $x_{n}$, one can note that they are slowly increasing, starting from $x_{2}=5$. Indeed, the bounds $5 \leq x_{n} \leq 9$ are readily established by induction on $n$ for $n \geq 3$. The log-convexity now follows by computing $\nabla R(n), \nabla S(n), \nabla R(n-1), \nabla S(n-1)$, and verifying the inequality (3.15) with $m=5, M=9$. To prove the log-balancedness of $\left(F_{n}^{(3)}\right)$, we start by computing

$$
\Delta_{R^{(3)}}(n)=\frac{(n-1)\left(7 n^{3}+7 n^{2}-n-2\right)}{n^{2}(n+1)^{2}}, \quad \Delta_{S^{(3)}}(n)=\frac{8\left(n^{4}-2 n^{3}-2 n^{2}+n+1\right)}{n^{2}(n+1)^{2}} .
$$

It is easy to check that these determinants are positive for $n \geq 3$, and that the conditions of Proposition 3.3 are valid for $n=2$.

Proof of the case $r=4$ is a bit more technical, but it flows along the same lines, and does not present any conceptual difficulties.

We now turn our attention to the recurrences with $S(n) \leq 0$. Such examples include, among others, Schröder numbers, Delannoy numbers and, more generally, sequences of values of Legendre polynomials. We start with a sequence closely connected with Franel numbers of order 3 .

The Apéry numbers, $\left(A_{n}\right)_{n \geq 0}$, given by the formula

$$
A_{n}=\sum_{k=0}^{n}\left(\begin{array}{l}
n \\
k
\end{array}\right)^{2}\left(\begin{array}{c}
n+k \\
k
\end{array}\right)^{2}=\sum_{k=0}^{n} \frac{[(n+k) !]^{2}}{(k !)^{4}[(n-k) !]^{2}}
$$

arose in Apéry's proof of irrationality of $\zeta(2)$ and $\zeta(3)$. They are connected with Franel numbers of order 3 via the identity

$$
A_{n}=\sum_{k=0}^{n}\left(\begin{array}{l}
n \\
k
\end{array}\right)\left(\begin{array}{c}
n+k \\
k
\end{array}\right) F_{k}^{(3)}, \quad n \geq 0
$$

(see [17] for history of this result). The first few Apéry numbers are 1,5,73, 1445, 33001, $819005, \ldots$.

Corollary 4.4. The sequence $A_{n}$ of Apéry numbers is log-balanced.

Proof. We start from the recurrence

$$
A_{n}=\frac{34 n^{3}-51 n^{2}+27 n-5}{n^{3}} A_{n-1}-\frac{(n-1)^{3}}{n^{3}} A_{n-2}, \quad n \geq 2,
$$


with initial conditions $A_{0}=1, A_{1}=5$ (see [4]). It is easy to prove by induction on $n$ that $x_{n} \geq 1$, that is, that the sequence of Apéry numbers is increasing. Hence we may take $m=1$ as the lower bound for $x_{n}$. Now the expression $\nabla R(n)+\nabla S(n)$ can serve as a lower bound for the expression (3.8), and the log-convexity of Apéry numbers follows from the inequality

$$
\nabla R(n)+\nabla S(n)=\frac{1}{n^{3}(n+1)^{3}}\left[50 n^{4}+52 n^{3}-10 n^{2}-12 n+4\right] \geq 0
$$

valid for all $n \geq 0$. For the rest, first note that $x_{3}=1445 / 73 \leq(3 / 2) x_{2}$, so we can take $n_{0}=2$. After computing $\Delta_{R}(n)$ and $\bar{\Delta}_{S}(n)$, we get

$$
\begin{aligned}
& \Delta_{R}(n)=\frac{34 n^{6}-72 n^{4}-28 n^{3}+27 n^{2}+7 n-5}{n^{3}(n+1)^{3}}, \\
& \bar{\Delta}_{S}(n)=\frac{(n-1)\left(n^{2}-n-1\right)\left(2 n^{3}+n^{2}-n-1\right)}{n^{3}(n+1)^{3}} .
\end{aligned}
$$

Both determinants are positive for $n \geq 2$, and the claim follows from Proposition 3.2.

Corollary 4.5. The sequence $r_{n}$ of large Schröder numbers is log-balanced.

Proof. Start from the recurrence

$$
r_{n}=\frac{3(2 n-1)}{n+1} r_{n-1}-\frac{n-2}{n+1} r_{n-2}, \quad n \geq 2
$$

with initial conditions $r_{0}=1, r_{1}=2$ (see [15]). By computing the first few values of $x_{n}=$ $r_{n} / r_{n-1}$, we guess the bounds $3 \leq x_{n} \leq 6$ and verify them by induction for all $n \geq 2$. The log-convexity of $\left(r_{n}\right)$ follows now by plugging the expressions $\nabla R(n)=9 /(n+1)(n+2)$ and $\nabla S(n)=-3 /(n+1)(n+2)$ in formula (3.8), together with $x_{n-1} \geq 3$. To prove the rest, we compute

$$
\Delta_{R}(n)=6 \frac{n-1}{n+2}, \quad \bar{\Delta}_{S}(n)=\frac{5-2 n}{n+2}
$$

and note that $\Delta_{R}(n) x_{n-1}+\bar{\Delta}_{S}(n) \geq 3 \Delta_{R}(n) x_{n-1}+\bar{\Delta}_{S}(n) \geq 0$ for all $n \geq 1$. Hence, by Proposition 3.4, the sequence $\left(r_{n}\right)$ is $\log$-balanced.

For combinatorial interpretations of $r_{n}$, the reader may wish to consult [15, Exercise 6.39].

Our next example is the sequence of values of Legendre polynomials in some fixed real $t \geq 1$.

Corollary 4.6. The sequence of values of Legendre polynomials $\left(P_{n}(t)\right)_{n \geq 0}$ is log-balanced for all real $t \geq 1$. 
Proof. We start from Bonnet recurrence:

$$
P_{n}(t)=\frac{2 n-1}{n} t P_{n-1}(t)-\frac{n-1}{n} P_{n-2}(t), \quad n \geq 2,
$$

with the initial conditions $P_{0}(t)=1, P_{1}(t)=t$. Passing to the recursion for the quotient sequence $x_{n}(t)=P_{n}(t) / P_{n-1}(t)$, we can easily establish the lower bound $x_{n}(t) \geq t$. By putting this lower bound, together with the expressions $\nabla R(n)=2 / n(n+1)$ and $\nabla S(n)=$ $-1 / n(n+1)$ in formula (3.8), we obtain the log-convexity of the sequence $\left(P_{n}(t)\right)_{n \geq 0}$. Further, by computing $\Delta_{R}(n)$ and $\bar{\Delta}_{S}(n)$, we get

$$
\Delta_{R}(n)=\frac{2 n^{2}-1}{n(n+1)}, \quad \bar{\Delta}_{S}(n)=\frac{-2 n^{2}+n+1}{n(n+1)} .
$$

If we suppose that $\Delta_{R}(n) x_{n-1}(t)+\bar{\Delta}_{S}(n)<0$ for some $n \geq 2$, we get $x_{n-1}(t)<(1 / t)\left(\left(2 n^{2}-\right.\right.$ $\left.n-1) /\left(2 n^{2}-1\right)\right)<1 / t<t$, in contradiction with $x_{n-1}(t) \geq x_{1}(t)=t$. Hence, the inequality $\Delta_{R}(n) x_{n-1}(t)+\bar{\Delta}_{S}(n) \geq 0$ holds for all $n \geq 2$, and the claim again follows from Proposition 3.4 .

By specializing the value of $t=3$, we get the sequence of central Delannoy numbers, $D_{n}=P_{n}(3)[15]$.

Corollary 4.7. The sequence $D_{n}$ of central Delannoy numbers is log-balanced.

The sequence $D_{n}$ counts the lattice paths from $(0,0)$ to $(n, n)$ using only the steps $(1,0)$, $(0,1)$, and $(1,1)$. Equivalently, it counts king paths from the lower left to the upper right corner of an $(n+1) \times(n+1)$ chess board.

In all examples considered so far, the sequence $\left(x_{n}\right)$ was increasing, but remained bounded. Our final example in this section shows that the same reasoning can be applied to the sequences whose quotient sequence increases unboundedly.

Corollary 4.8. Let $\left(a_{n}\right)$ be the sequence counting directed column-convex polyominoes of height $n$. (See [3] for the definition of these objects.) The sequence $\left(a_{n}\right)$ is log-balanced.

Proof. From the recurrence

$$
a_{n+1}=(n+1) a_{n}+a_{1}+a_{2}+\cdots+a_{n}, \quad n \geq 3,
$$

with initial conditions $a_{1}=1, a_{2}=3$, given in [3], one can easily obtain the two-term recurrence

$$
a_{n}=(n+2) a_{n-1}-(n-1) a_{n-2}, \quad n \geq 3,
$$

with $a_{1}=1, a_{2}=3$. It can easily be shown by induction on $n$ that the sequence $x_{n}=$ $a_{n} / a_{n-1}$ is interlaced with the sequence $b_{n}=n+1$, that is, that $n+1 \leq x_{n} \leq n+2$. Hence the sequence $\left(x_{n}\right)$ is increasing, and $\left(a_{n}\right)$ is log-convex. Taking $R(n)=n+2, S(n)=-n+1$, we get $\Delta_{R}(n)=2, \bar{\Delta}_{S}(n)=1-n$. Suppose that $\Delta_{R}(n) x_{n-1}(t)+\bar{\Delta}_{S}(n)<0$ for some $n \geq 3$. It follows that $x_{n-1}<(n-1) / 2$, contradicting the interlacing of $x_{n}$ and $b_{n}$. The claim now follows by checking the base of induction, that is, that $x_{3}=13 / 3 \leq(3 / 2) \cdot 3=(3 / 2) x_{2}$. 


\section{Further developments}

The method exposed in Section 3 can be extended to the sequences given by a three- (or more-) term recurrence in a straightforward way. As an illustration, we treat here the case when all coefficient functions are positive and increasing.

Let $\left(a_{n}\right)$ be a sequence of positive real numbers given by the recurrence

$$
a_{n}=R(n) a_{n-1}+S(n) a_{n-2}+T(n) a_{n-3}, \quad n \geq 3,
$$

with given initial conditions $a_{0}, a_{1}$, and $a_{2}$. Then the recurrence for the quotient sequence is given by

$$
x_{n}=R(n)+\frac{S(n)}{x_{n-1}}+\frac{T(n)}{x_{n-1} x_{n-2}}
$$

for $n \geq 3$. We suppose inductively that $x_{n_{0}} \leq x_{n_{0}+1} \leq \cdots \leq x_{n}$ for some $n_{0} \in \mathbb{N}$, and we want to find sufficient conditions for $x_{n+1} \geq x_{n}$. This inequality can be stated as

$$
R(n+1)+\frac{S(n+1)}{x_{n}}+\frac{T(n)}{x_{n} x_{n-1}}-R(n)-\frac{S(n)}{x_{n-1}}-\frac{T(n)}{x_{n-1} x_{n-2}} \geq 0,
$$

or equivalently

$$
x_{n} x_{n-1} x_{n-2} \nabla R(n)+x_{n-2}\left[x_{n-1} S(n+1)-x_{n} S(n)\right]+x_{n-2} T(n+1)-x_{n} T(n) \geq 0 .
$$

Now we proceed by a sequence of strengthenings of this inequality, leading to a sufficient condition that will be expressed in quantities known and reasonably easy to check. First we replace $S(n+1)$ and $T(n+1)$ by $S(n)$ and $T(n)$, respectively. This yields

$$
x_{n} x_{n-1} x_{n-2} \nabla R(n)+x_{n-2} S(n)\left(x_{n-1}-x_{n}\right)+T(n)\left(x_{n-2}-x_{n}\right) \geq 0 .
$$

By adding $x_{n-1}-x_{n-1}$ to the term $x_{n-2}-x_{n}$ and grouping the terms accordingly, we obtain

$$
x_{n} x_{n-1} x_{n-2} \nabla R(n)+\left[x_{n-2} S(n)+T(n)\right]\left(x_{n-1}-x_{n}\right)+T(n)\left(x_{n-2}-x_{n-1}\right) \geq 0 .
$$

We now look more closely at the term $x_{n-1}-x_{n}$. By inductive hypothesis, it must be nonpositive, but we do not have any information about its magnitude. Expressing $x_{n-1}$ and $x_{n}$ via recurrence $(5.2)$ yields

$$
\begin{aligned}
x_{n-1}-x_{n}= & -\nabla R(n-1)+\frac{1}{x_{n-1} x_{n-2}}\left[x_{n-1} S(n-1)-x_{n-2} S(n)\right] \\
& +\frac{1}{x_{n-1} x_{n-2} x_{n-3}}\left[x_{n-1} T(n-1)-x_{n-3} T(n)\right] .
\end{aligned}
$$

By replacing $x_{n-1}$ in the first square brackets on the right-hand side of the above relation by $x_{n-2}$, and in the second square brackets by $x_{n-3}$, one obtains the following inequality:

$$
x_{n-1}-x_{n} \geq-\nabla R(n-1)-\frac{1}{x_{n-1}} \nabla S(n-1)-\frac{1}{x_{n-1} x_{n-2}} \nabla T(n-1) .
$$


Similarly,

$$
x_{n-2}-x_{n-1} \geq-\nabla R(n-2)-\frac{1}{x_{n-2}} \nabla S(n-2)-\frac{1}{x_{n-2} x_{n-3}} \nabla T(n-2) .
$$

Plugging in formulae (5.8) and (5.9) in (5.6), we obtain the inequality

$$
\begin{aligned}
& x_{n} x_{n-1} x_{n-2} \nabla R(n) \\
& \geq {\left[x_{n-2} S(n)+T(n)\right]\left[\nabla R(n-1)+\frac{1}{x_{n-1}} \nabla S(n-1)+\frac{1}{x_{n-1} x_{n-2}} \nabla T(n-1)\right] } \\
& \quad+T(n)\left[\nabla R(n-2)+\frac{1}{x_{n-2}} \nabla S(n-2)+\frac{1}{x_{n-2} x_{n-3}} \nabla T(n-2)\right] .
\end{aligned}
$$

Finally, by replacing the values of $x_{n}, x_{n-1}, x_{n-2}$, and $x_{n-3}$ by their lower and upper bounds, we arrive at the following inequality:

$$
\begin{aligned}
m^{3} \nabla R(n) \geq & {[M \cdot S(n)+T(n)]\left[\nabla R(n-1)+\frac{1}{m} \nabla S(n-1)+\frac{1}{m^{2}} \nabla T(n-1)\right] } \\
& +T(n)\left[\nabla R(n-2)+\frac{1}{m} \nabla S(n-2)+\frac{1}{m^{2}} \nabla T(n-2)\right] .
\end{aligned}
$$

Obviously, inequality (5.11) implies inequality (5.6), and this one, in turn, implies our initial inequality $x_{n+1} \geq x_{n}$. Hence, inequality (5.11) provides a sufficient condition of log-convexity for the sequence $\left(a_{n}\right)$.

Now, assuming the log-convexity of $\left(a_{n}\right)$, by following the same reasoning as in the proof of Proposition 3.3, we obtain sufficient conditions of log-balancedness of $\left(a_{n}\right)$ in the form

$$
\Delta_{R}(n) x_{n-1} x_{n-2}+\Delta_{S}(n) x_{n-2}+\Delta_{T}(n) \geq 0
$$

where $\Delta_{R}(n)$ and $\Delta_{S}(n)$ are as before, and $\Delta_{T}(n)$ is defined analogously.

As an illustration of this result, we prove that the sequence $\left(R_{n}\right)$, counting the Baxter permutations of size $n$, is log-balanced. (See [15, page 246 and pages 278-279] for more details on Baxter permutations.) The numbers $R_{n}$ satisfy a third-order linear recurrence with the coefficient functions given by

$$
\begin{aligned}
& R(n)=2 \frac{9 n^{3}+3 n^{2}-4 n+4}{(n+2)(n+3)(3 n-2)}, \\
& S(n)=\frac{(3 n-1)(n-2)\left(15 n^{2}-5 n-14\right)}{(n+1)(n+2)(n+3)(3 n-2)}, \\
& T(n)=8 \frac{(3 n+1)(n-2)^{2}(n-3)}{(n+1)(n+2)(n+3)(3 n-2)} .
\end{aligned}
$$


With a bit of help from a computer algebra system such as, for example, Mathematica, it can be proved that $7 \leq x_{n} \leq 9$ for $n \geq 47$. Verifying the inequality (5.11) then boils down to checking that a certain rational function of $n$ (with the degrees of the numerator and denominator equal to 12 and 14, resp.) is nonnegative for sufficiently large values of the argument. By substituting $n+3$ in place of $n$, it becomes obvious that all the coefficients become positive, and hence, the function cannot change the sign for $n \geq 3$. The increasing behavior of $x_{n}$ for $n \leq 47$ is easily checked by direct computation. Hence the sequence $\left(R_{n}\right)$ is log-convex. To prove the log-balancedness, it is easy to check that all three determinants

$$
\begin{aligned}
& \Delta_{R}(n)=\frac{27 n^{5}+18 n^{4}+3 n^{3}+76 n^{2}+100 n+16}{(n+1)(n+2)(n+3)(n+4)(3 n+1)(3 n-2)}, \\
& \Delta_{S}(n)=\frac{135 n^{5}-990 n^{4}+87 n^{3}+1036 n^{2}+4 n-112}{(n+1)(n+2)(n+3)(n+4)(3 n+1)(3 n-2)}, \\
& \Delta_{T}(n)=\frac{9 n^{5}-138 n^{4}+349 n^{3}-80 n^{2}-252 n-48}{(n+1)(n+2)(n+3)(n+4)(3 n+1)(3 n-2)}
\end{aligned}
$$

are positive for $n \geq 13$, and the log-balancedness of $\left(R_{n}\right)$ follows by directly verifying defining inequalities in the remaining cases. All the Mathematica calculations necessary for verifying the above inequalities were performed exactly.

The scope of our approach can also be extended in another direction, namely, to linear nonhomogeneous recurrences. Here we indicate, after the fashion of [9], how such recursions can be transformed in a form suitable for application of our method. So, for example, let $\left(a_{n}\right)$ be given by a linear nonhomogeneous recurrence of the first order:

$$
a_{n}=R(n) a_{n-1}+S(n)
$$

with the initial condition $a_{0}$. By writing down the recurrence (5.15) for successive indices, multiplying and subtracting as to cancel the nonhomogeneous part, one obtains the homogeneous second-order linear recurrence for $a_{n}$ :

$$
a_{n}=\left[R(n)+\frac{S(n)}{S(n-1)}\right] a_{n-1}-\frac{R(n-1) S(n)}{S(n-1)} a_{n-2} .
$$

By denoting $R^{*}(n)=R(n)+(S(n) / S(n-1)), S^{*}(n)=-(R(n-1) S(n) / S(n-1))$, and dividing through by $a_{n-1}$, we get a recurrence for $x_{n}$ of the type (3.2) and the further treatment depends on the combination of signs of $R^{*}(n)$ and $S^{*}(n)$.

Similarly, for a second-order linear recurrence

$$
a_{n}=R(n) a_{n-1}+S(n) a_{n-2}+T(n)
$$

we obtain

$$
x_{n}=R(n)+\frac{S(n)}{x_{n-1}}+\frac{T(n)}{T(n-1)}\left[1-\frac{R(n-1)}{x_{n-1}}-\frac{S(n-1)}{x_{n-1} x_{n-2}}\right] .
$$

Then we can proceed as before. 
Finally, a word of caution. It would be hasty to conclude, from the cited examples, that all combinatorially interesting sequences are log-balanced. For example, the sequences $a_{n}=(n !)^{2}, a_{n}=(n-1) !$, and $a_{n}=\sum_{k=0}^{n} k$ ! are not log-balanced, since their quotient sequences grow too fast. It is also interesting to note that the property of log-balancedness is not shift-invariant; one can easily see that the sequence $(n+1)$ ! is log-balanced, while $(n-1)$ ! is not.

One could, in principle, consider an alternative approach to the question of logbalancedness, that is, in a sense dual to ours. One could take a log-concave sequence $\left(a_{n}\right)$ and ask for the sufficient conditions for the sequence $\left(n ! a_{n}\right)$ to be log-convex. Since it appears that the log-convex sequences are much more common among the sequences of combinatorial interest, we will not pursue this alternative approach here.

\section{Acknowledgment}

The author acknowledges the support of the Welch Foundation of Houston, Texas, via Grant no. BD-0894.

\section{References}

[1] M. Aigner, Motzkin numbers, European J. Combin. 19 (1998), no. 6, 663-675.

[2] N. Asai, I. Kubo, and H.-H. Kuo, Bell numbers, log-concavity, and log-convexity, Acta Appl. Math. 63 (2000), 79-87.

[3] E. Barcucci, A. Del Lungo, R. Pinzani, and R. Sprugnoli, La hauter des polyominos dirigés verticalement convexe [The height of directed column-convex polyominoes], Sém. Lothar. Combin. 31 (1993), no. B31d, 1-11 (French).

[4] F. Beukers, Another congruence for the Apéry numbers, J. Number Theory 25 (1987), no. 2, 201210.

[5] F. Brenti, Unimodal, Log-Concave and Pólya Frequency Sequences in Combinatorics, American Mathematical Society, Rhode Island, 1989.

[6] D. Callan, Notes on Motzkin and Schröder numbers, preprint, 2000.

[7] E. Deutsch and L. Shapiro, A survey of the Fine numbers, Discrete Math. 241 (2001), no. 1-3, 241-265.

[8] T. Došlić, Some new inequalities for Motzkin numbers, Math. Inequal. Appl. 5 (2002), no. 2, 171-174.

[9] T. Došlić and D. Veljan, Logarithmic behavior of some combinatorial sequences, submitted to Discrete Mathematics.

[10] S. Karlin, Total Positivity. Vol. I, Stanford University Press, California, 1968.

[11] H.-H. Kuo, White Noise Distribution Theory, Probability and Stochastics Series, CRC Press, Florida, 1996.

[12] K. A. Penson and A. I. Solomon, Coherent states from combinatorial sequences, Quantum Theory and Symmetries (Kraków, 2001), World Scientific Publishing, New Jersey, 2002, pp. 527-530.

[13] B. Sagan, Inductive and injective proofs of log concavity results, Discrete Math. 68 (1988), no. 2-3, 281-292.

[14] R. P. Stanley, Log-concave and unimodal sequences in algebra, combinatorics, and geometry, Graph Theory and Its Applications: East and West (Jinan, 1986), Ann. New York Acad. Sci., vol. 576, New York Academy of Sciences, New York, 1989, pp. 500-535.

[15] - Enumerative Combinatorics. Vol. 2, Cambridge Studies in Advanced Mathematics, vol. 62, Cambridge University Press, Cambridge, 1999. 


\section{Log-balanced combinatorial sequences}

[16] - Positivity problems and conjectures in algebraic combinatorics, Mathematics: Frontiers and Perspectives (V. Arnold, M. Atiyah, P. Lax, and B. Mazur, eds.), American Mathematical Society, Rhode Island, 2000, pp. 295-319.

[17] V. Strehl, Recurrences and Legendre transform, Sém. Lothar. Combin. 29 (1992), no. B29b, 1-22.

Tomislav Došlić: Department of Informatics and Mathematics, Faculty of Agriculture, University of Zagreb, Svetošimunska 25, 10000 Zagreb, Croatia

Current address: Department of Marine Sciences, Texas A\&M University at Galveston, Galveston, Texas, TX 77551, USA

E-mail address: doslic@math.hr 


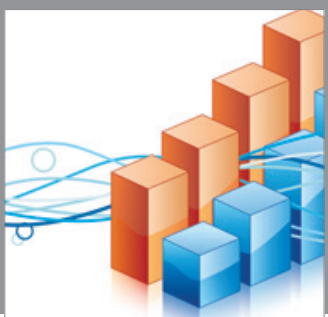

Advances in

Operations Research

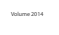

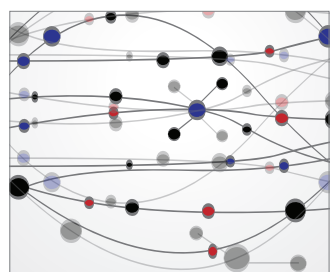

\section{The Scientific} World Journal
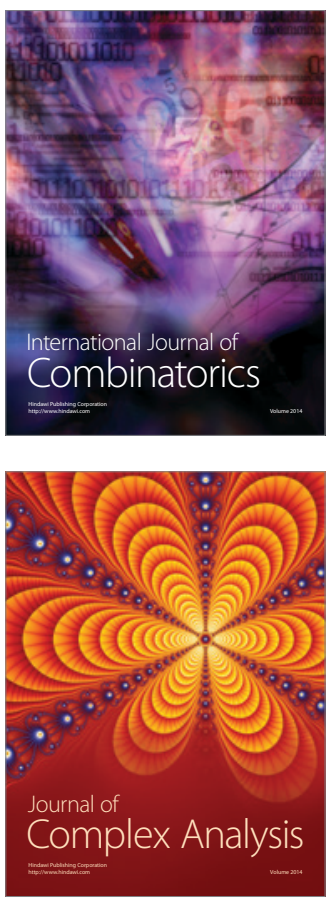

International Journal of

Mathematics and

Mathematical

Sciences
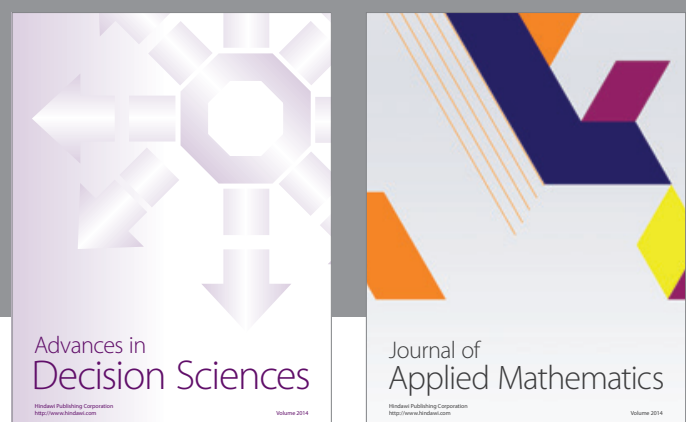

Journal of

Applied Mathematics
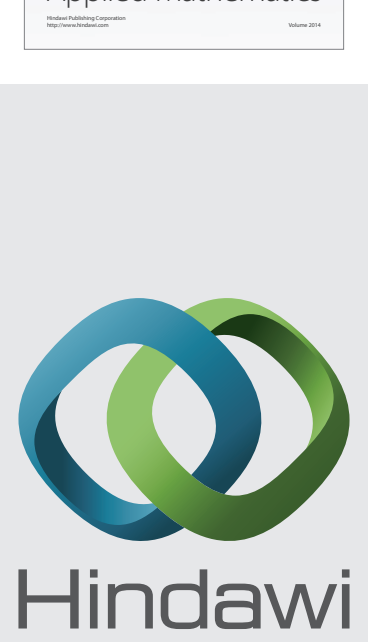

Submit your manuscripts at http://www.hindawi.com
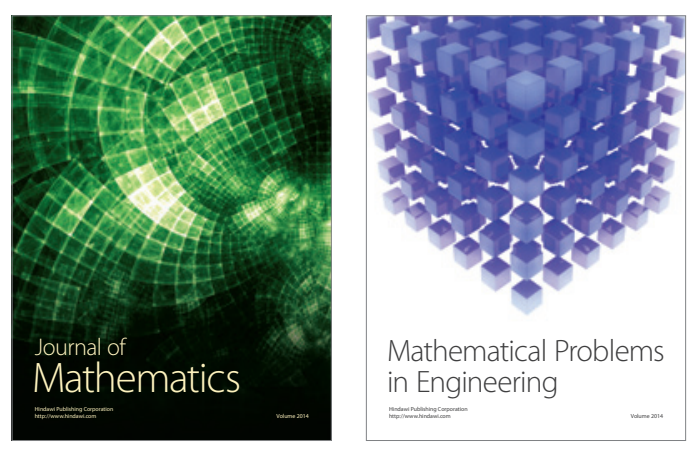

Mathematical Problems in Engineering
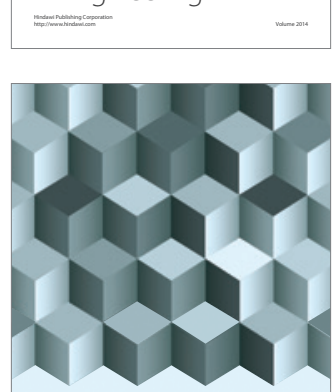

Journal of

Function Spaces
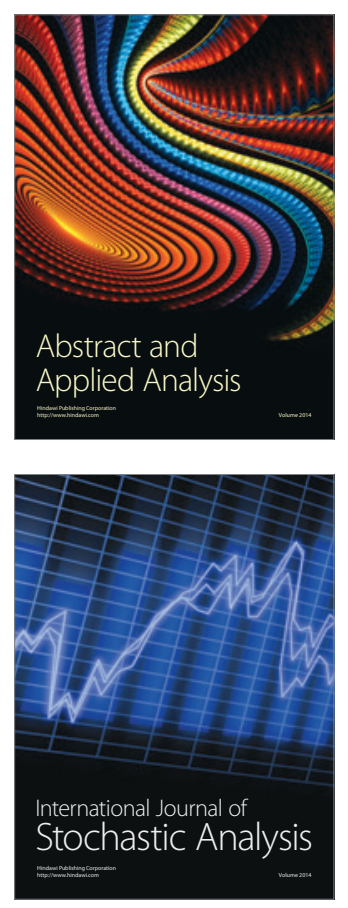

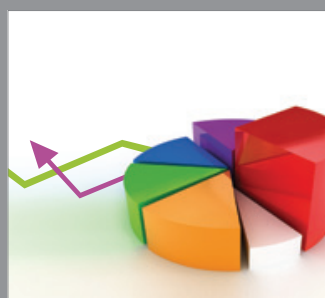

ournal of

Probability and Statistics

Promensencen
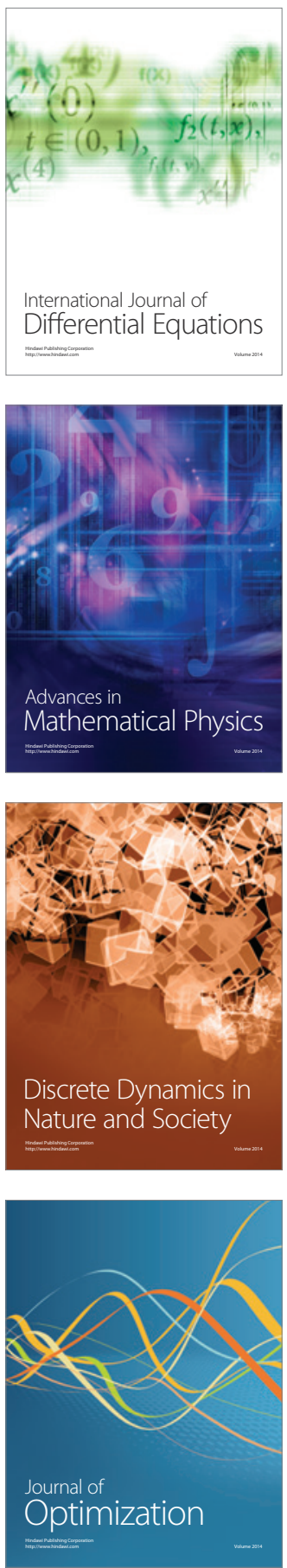